Sharif University of Technology
Scientia Iranica
SCIENTIA
I RAN ICA

Research Note

\title{
Modeling and multivariable active disturbance rejection control of a hydraulic looper multivariable system
}

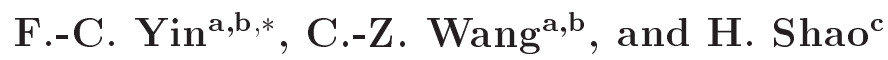 \\ a. Institute of Manufacturing Engineering, HuaQiao University, Xiamen 361021, China. \\ b. Fujian Engineering Research Center of Intelligent Manufacturing for Brittle Materials, Fujian 361021 , China. \\ c. College of Information Science and Engineering, HuaQiao University, Xiamen 361021, China.
}

Received 19 August 2018; received in revised form 14 March 2018; accepted 18 August 2018

\section{KEYWORDS}

Hot strip mill;

Hydraulic looper system;

Mathematical model; Multivariable active disturbance rejection control;

MATLAB/Simulink.

\begin{abstract}
Controlling the looper height and strip tension is important in hot strip mills, because these variables affect both the strip quality and strip threading. Many researchers have proposed and applied a variety of control schemes for this problem; however, the increasingly strict market demand for strip quality requires further improvements. This paper describes a Multivariable Active Disturbance Rejection Control (MADRC) strategy that realizes the decoupling control of a hydraulic looper multivariable system. Simulation experiments of a traditional Proportion-Integration (PI) controller and the proposed MADRC controller were conducted using MATLAB/Simulink software. The simulation results show that the proposed MADRC ensures good robustness and adaptability under modeling uncertainty and external disturbance. It is concluded that the designed MADRC controller produces better dynamic performance than the traditional PI controller does, and the proposed looper control system is effective and practical.
\end{abstract}

(C) 2018 Sharif University of Technology. All rights reserved.

\section{Introduction}

A hot strip mill rolls cast steel slabs into thin sheets $[1,2]$. A typical hot strip mill facility is composed of the following units: reheat furnace, roughing mill, transfer table, coilbox, crop shear, finishing mill, run-out table, and coiler [3,4]. Bars with thickness of around $250 \mathrm{~mm}$ are reheated to a temperature of approximately $1200^{\circ} \mathrm{C}$ in the reheat furnace. In the roughing mill, the reheated slabs are reduced to thickness of 25-50 mm. The resulting sheet bar is then transported to the finishing mill, where it is further reduced to the final thickness of $0.8-20 \mathrm{~mm}$.

\footnotetext{
* Corresponding author. Tel.: +86-18159284929 E-mail addresses: yfc_ral@163.com (F.-C. Yin); 284712425@qq.com (C.-Z.Wang);84185803@qq.com (H. Shao)
}

doi: $10.24200 /$ sci. 2018.5040 .1056
During the hot strip rolling process, the looper angle and strip tension control play an important role in both the dimensional quality and mass flow of the strips $[5,6]$. The control target is to keep the looper angle and strip tension as close to the desired values as possible. For the past three decades, hydraulic loopers have been widely used in the steel industry because of their fast transient response and high stability and precision [7]. However, there are several disadvantages to them, such as significant uncertainties and disturbances in system parameter and a nonlinear hydraulic servo system, which complicates the design of the hydraulic looper control system [8,9]. A number of scholars have investigated solutions to this problem. Wang [10] presented the guaranteed cost-sliding mode control system that gives the looper system a fast response time. Zhong et al. [11] studied the looper system controller with an almost disturbance decoupling control strategy to enhance the looper system's robustness. Pittner et 
al. [12] proposed an optimal controller based on a statedependent Riccati equation technique to deal with the unmodeled dynamics and large parameter variations of looper system. Active disturbance rejection control, which is well known for its robustness, has been used to design the controller in many industrial fields. For instance, Wang et al. [13] suggested a practical active disturbance rejection control solution in the monitoring Automatic Gauge Control (AGC) system of hot strip mill, and Zou et al. [14] designed a controller with active disturbance rejection control theory for hydraulic width control system in rough mills. Huang et al. [15] designed the control circuit of inertial platform with active disturbance rejection control theory. Su and Qiu [16] introduced the active disturbance rejection controller into robot uncalibrated visual servoing system. Dong et al. [17] introduced the active disturbance rejection controller into electric power assist steering system. In this paper, for hydraulic looper system, the interaction between looper height and strip tension is considered, and a Multivariable Active Disturbance Rejection Control (MADRC) strategy is realized by combining ADRC static decoupling technology with ESO dynamic decoupling technology. The resulting controller can be applied in both the looper height and strip tension closed loops based on the coupled transfer function model of the hydraulic looper system.

\section{Model of hydraulic looper system}

In this section, an overview of the looper height and strip tension model is presented to analyze hydraulic looper systems. The model description closely follows that in [1] and forms the background of the control problem. Figure 1 shows the geometry of the looper and strip for the adjacent stands of seven rolling stands in the hot strip finishing mill.

In Figure 1, $\theta$ is the looper angle, $L$ is the distance between two stands, $L_{2}$ is the distance between the actual pass line and the looper pivot, $L_{3}$ is the distance between stand and the looper pivot, $r$ is the radius of the looper roll, $\delta$ is the angle between the looper power arm and the movable arm, $\phi$ is the angle between the hydraulic cylinder and the vertical direction, $\xi$ is the angle between the tangential direction of the looper power arm and the hydraulic cylinder, $\nu_{i+1}$ is the roll line speed at the $(i+1)$ th stand, $\nu_{i}$ is the roll line speed at the $i$ th stand, $\nu_{\text {out }}^{i}$ is the delivery speed of the strip at the $i$ th stand, and $\nu_{\mathrm{in}}^{i+1}$ is the entry speed at the $(i+1)$ th stand.

\subsection{Model of strip tension system}

Strip tension increment $\Delta \sigma$ is approximately proportional to both the Young's modulus and the strip stretch according to the following equation:

$$
\Delta \sigma=\frac{E}{L}\left(\Delta L+\Delta \delta_{v}\right),
$$

where $\Delta L$ is the accumulated loop length, $E$ is the Young's modulus, and $\Delta \delta_{v}$ is the looper variation caused by changes in speed.

According to the geometrical relationship in Figure 1 , the relationship between the looper height and looper angle is:

$$
\Delta L=E G+G F-L
$$

where:

$$
\begin{aligned}
& E G=\sqrt{\left[L_{3} \sin (\theta)-L_{2}+r\right]^{2}+\left[L_{3} \cos (\theta)+L_{3}\right]^{2}}, \\
& G F=\sqrt{\left[L_{3} \sin (\theta)-L_{2}+r\right]^{2}+\left[L-L_{3}-L_{3} \cos (\theta)\right]^{2}},
\end{aligned}
$$

and $\Delta \delta_{v}$ can be calculated as follows:

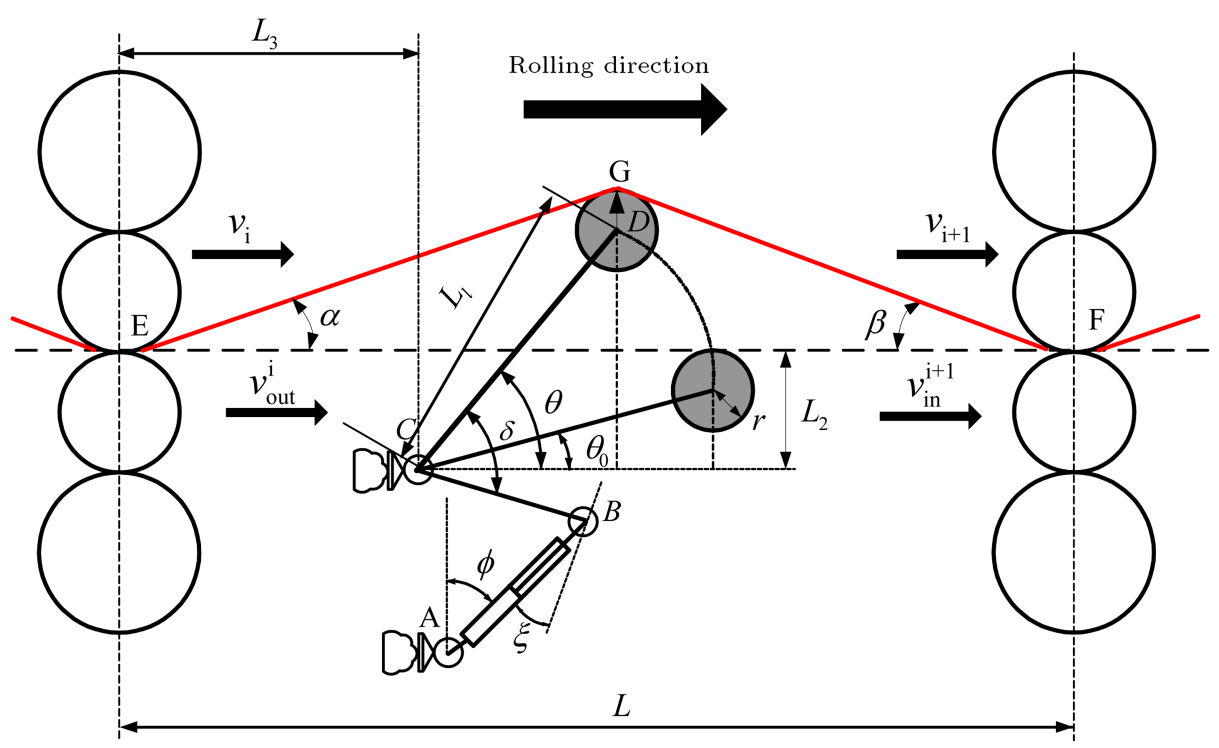

Figure 1. Schematic drawing of a hydraulic looper system. 


$$
\Delta \delta_{v}=\int{ }_{t_{0}}^{t}\left(\Delta \nu_{\mathrm{in}}^{i+1}-\Delta \nu_{\mathrm{out}}^{i}\right) d t
$$

for:

$$
\begin{aligned}
\Delta \nu_{\mathrm{in}}^{i+1} & -\Delta \nu_{\mathrm{out}}^{i}=-\Delta \beta_{i+1} \nu_{i+1}-\left(1+f_{i}\right) \Delta \nu_{i} \\
& -\Delta f_{i} \nu_{i},
\end{aligned}
$$

where $\beta_{i+1}$ is the backward slip at the $(i+1)$ th stand, and $f_{i}$ is the forward slip at the $i$ th stand.

By substituting Eqs. (2) and (4) into Eq. (1), the model is approximately linearized using a Taylor expansion, which gives:

$$
\begin{aligned}
s \Delta \sigma= & \frac{E}{L}\left[\frac{d L}{d \theta} \Delta \theta-\left(\nu_{i} \frac{\partial f_{i}}{\partial \sigma}+\nu_{i+1} \frac{\partial \beta_{i+1}}{\partial \sigma}\right) \Delta \sigma\right. \\
& \left.-\left(1+f_{i}\right) \Delta \nu_{i}\right] .
\end{aligned}
$$

\subsection{Model of looper height system}

By applying Newton's law of motion to the hydraulic looper system, the following equation can be obtained:

$$
J \cdot \ddot{\theta}=M \cdot \cos (\delta-\phi-\theta)-L_{l} \cdot F_{\text {load }},
$$

where $M$ is the output torque of the looper hydraulic cylinder, $F_{\text {load }}$ is the total load acting on the looper, and $L_{l}$ is the length of the movable arm.

$F_{\text {load }}$ is composed of three main components: strip tension, $F_{\sigma}$, total force of the strip weight and looper weight, $F_{w}$, and strip bending force, $F_{b}$ :

$$
F_{\text {load }}=F_{\sigma}+F_{w}+F_{b},
$$

where the above three quantities are modeled as follows. $F_{\sigma}$ can be evaluated as:

$$
F_{\sigma}=\sigma \cdot W \cdot H \cdot[\sin (\theta+\beta)-\sin (\theta-\alpha)],
$$

where $W$ is the strip width, and $H$ is the strip exit thickness. $F_{w}$ can be evaluated as follows:

$$
F_{w}=\left(\frac{1}{2} W_{S} \cdot g+W_{R} \cdot g\right) \cdot \cos \theta,
$$

where $W_{R}$ is the looper weight, and $W_{R}$ is the looper arm weight. $F_{b}$ can be evaluated as follows:

$$
F_{b}=\left[16 E \cdot W \cdot H \cdot\left(\frac{H}{L}\right)^{3}\right] \cos \theta .
$$

By substituting Eq. (7) into Eq. (6), the model is approximately linearized using a Taylor expansion, which gives:

$$
J s^{2} \Delta \theta=\Delta M-\frac{\partial M}{\partial \theta} \Delta \theta-\frac{\partial M}{\partial \sigma} \Delta \sigma .
$$

\subsection{Actuator modeling}

The actuator of the hydraulic looper is a hydraulic cylinder that is driven by a servo valve. The natural frequency of the hydraulic cylinder is higher than $20 \mathrm{~Hz}[13,14]$. The transfer function of the servo valve can thus be described as follows:

$$
G_{s v}(s)=\frac{Q_{v}}{i}=\frac{K_{s v}}{\frac{s^{2}}{\omega_{s v}^{2}}+2 \frac{\xi_{s v}}{\omega_{s v}} s+1},
$$

where $Q_{v}\left(\mathrm{~m}^{2} / \mathrm{s}\right)$ is the flow rate of the servo valve, $i$ (A) is the control current of the servo valve, $K_{s v}$ $\left(\mathrm{m}^{3} / \mathrm{s} / \mathrm{A}\right)$ is the flow gain coefficient of the servo valve, $\omega_{s v}(\mathrm{rad} / \mathrm{s})$ is the natural frequency, and $\xi_{s v}$ is the damping coefficient.

Based on the balance equation of the hydraulic cylinder and the load force and neglecting the damping coefficient and leakage coefficient, the transfer function of hydraulic cylinder, $G_{s}(s)$, can be obtained as follows:

$$
G_{s}(s)=\frac{F}{Q_{v}}=\frac{4 A \beta_{e}\left(M_{e} s^{2}+K\right)}{4 A^{2} \beta_{e} s+M_{e} V_{t} s^{3}+K V_{t} s},
$$

where $F(\mathrm{~N})$ is the thrust of the hydraulic cylinder, $A\left(\mathrm{~m}^{2}\right)$ is the effective area of the hydraulic cylinder piston, $\beta_{e}\left(\mathrm{~N} / \mathrm{m}^{2}\right)$ is the elastic modulus of hydraulic oil, $K(\mathrm{~N} / \mathrm{m})$ is the equivalent load stiffness, $M_{e}(\mathrm{~kg})$ is the equivalent quality of the load, and $V_{t}\left(\mathrm{~m}^{3}\right)$ is the maximum volume of the cylinder cavity that is under pressure.

The transfer function of the rolling mill's main drive system, $G_{v}(s)$, can be described by a first-order system:

$$
G_{v}(s)=\frac{1}{T_{v} s+1},
$$

where $T_{v}$ is the time constant.

\subsection{Transfer function model of hydraulic looper system}

A block diagram of the linearized model is given in Figure 2. The manipulated variables include roll velocity, $\nu$, and control current, $i$. The controlled variables include interstand tension $\sigma$ and looper angle $\theta$.

The coupling transfer function model can be obtained by a Laplace transform as follows:

$$
\left[\begin{array}{l}
\theta(s) \\
\sigma(s)
\end{array}\right]=\left[\begin{array}{ll}
G_{v \theta}(s) & G_{i \theta}(s) \\
G_{v \sigma}(s) & G_{i \sigma}(s)
\end{array}\right] \cdot\left[\begin{array}{c}
\nu(s) \\
i(s)
\end{array}\right] .
$$

The No. 5 stand, No. 6 stand, and No. 5 hydraulic looper in the $1700 \mathrm{~mm}$ hot strip mill were taken as the research objects in a simulation. The initial value of the looper angle was set to $0.296 \mathrm{rad}$, and the initial value of the strip tension was set to $5 \mathrm{MPa}$. The simulation parameters are given in Table 1.

Using the above data, the coupled transfer functions can be obtained by Eqs. (16) to (19) as shown in Box I. 


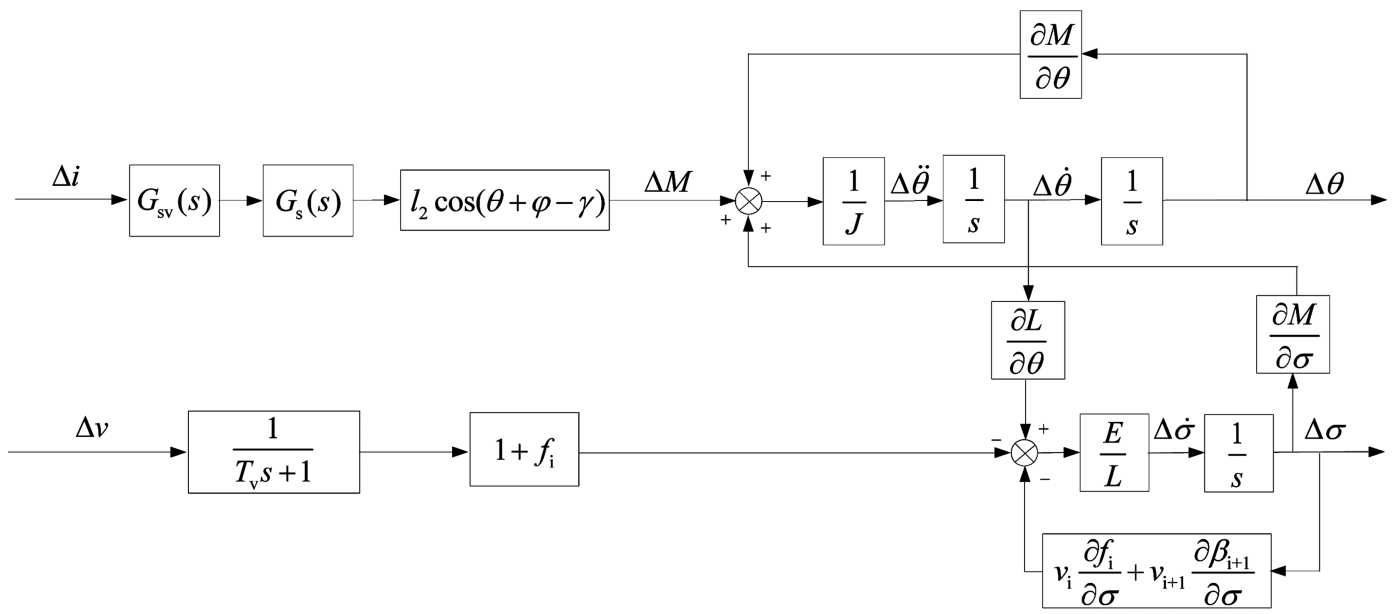

Figure 2. Linear model of a hydraulic looper system.

$$
\begin{aligned}
& G_{v \theta}(s)=\frac{0.1542 e 18+0.189 e 20 s^{3}+0.1523 e 21 s^{2}+0.756 e 22 s+0.3626 e 23}{\left(3.3 e 4 s^{9}+1.604 e 8 s^{8}+8.38 e 10 s^{7}+6.715 e 13 s^{6}+2.697 e 16 s^{5}\right.}\left.+2.712 e 18 s^{4}+1.1 e 20 s^{3}+5.346 e 20 s^{2}+2.451 e 20 s\right) \\
& \\
& G_{i \theta}(s)=\frac{0.7135 e 10 s^{4}+0.338 e 14 s^{3}+0.1392 e 17 s^{2}+0.1262 e 20 s+0.4206 e 22}{\left(3.3 e 4 s^{8}+1.604 e 8 s^{7}+8.38 e 10 s^{6}+6.715 e 13 s^{5}+2.697 e 16 s^{4}\right.} \\
&\left.\quad+2.712 e 18 s^{3}+1.1 e 20 s^{2}+5.346 e 20 s+2.451 e 20\right) \\
& G_{v \sigma}(s)=\frac{0.4872 e 27 s^{3}+0.2436 e 28 s^{2}+0.1949 e 30 s+0.9745 e 30}{\left(3.3 e 4 s^{8}+1.604 e 8 s^{7}+8.38 e 10 s^{6}+6.715 e 13 s^{5}+2.697 e 16 s^{4}\right.} \\
&\left.+2.712 e 18 s^{3}+1.1 e 20 s^{2}+5.346 e 20 s+2.451 e 20\right)
\end{aligned}
$$

Box I

\section{Looper control architecture}

\subsection{Traditional PI control scheme}

The classical control scheme for the hydraulic looper is based on a Proportional-Integral (PI) control architecture, as depicted in Figure 3. Strip tension error, $\Delta \sigma$, is controlled by a PI regulator that acts, with a trim, on the reference control current, $i_{\text {ref }}$, of the servo valve. Looper angle deviation, $\Delta \theta$, is regulated by a control loop based on PI controller that acts, with a trim, on reference speed, $\nu_{\text {ref }}[15,16]$.

The above PI control architecture is widely used mainly because of its simplicity. However, this control scheme can perform very poorly in the presence of disturbances and modeling uncertainties. For example, if the looper angle cannot be maintained near the desired value, the dynamic equilibrium of the metal mass flow will be broken. Hence, in the following, a multivariable active disturbance rejection control scheme will be proposed.

\subsection{Active disturbance rejection control: a brief summary}

Han [17] firstly put forward the active disturbance rejection control theory. Compared with PID control theory, it overcomes the differential obsession by 
Table 1. Simulation parameters of a hydraulic looper system.

\begin{tabular}{llll}
\hline \multicolumn{1}{c}{ Variable } & Value & \multicolumn{1}{c}{ Variable } & \multicolumn{1}{c}{ Value } \\
\hline$W(\mathrm{~m})$ & 1.265 & $L_{2}(\mathrm{~m})$ & 0.155 \\
$H(\mathrm{~m})$ & 0.00407 & $T_{v}(\mathrm{~s})$ & 0.0885 \\
$E(\mathrm{GPa})$ & 150 & $A\left(\mathrm{~m}^{2}\right)$ & 0.001 \\
$\delta(\mathrm{rad})$ & 0.75 & $M_{e}(\mathrm{~kg})$ & 500 \\
$L(\mathrm{~m})$ & 5.8 & $V_{t}\left(\mathrm{~m}^{3}\right)$ & 0.005 \\
$R(\mathrm{~m})$ & 0.61 & $\beta_{e}\left(\mathrm{~N} / \mathrm{m}^{2}\right)$ & 1187 \\
$r(\mathrm{~m})$ & 0.092 & $K\left(\mathrm{~m}^{5} / \mathrm{N} . \mathrm{s}\right)$ & $1.9964 \times 10^{5}$ \\
$J\left(\mathrm{~kg} . \mathrm{m}^{2}\right)$ & 480 & $K_{s v}\left(\mathrm{~m}^{5} / \mathrm{N} . \mathrm{s}\right)$ & $3.3 \times 10^{11}$ \\
$W_{R}(\mathrm{~kg})$ & 370 & $\zeta_{s v}$ & 0.6 \\
$W_{s}(\mathrm{~kg})$ & 190 & $\nu_{5}(\mathrm{~m} / \mathrm{s})$ & 5.61 \\
$H(\mathrm{~m})$ & 0.48 & $\nu_{6}(\mathrm{~m} / \mathrm{s})$ & 6.86 \\
$H_{1}(\mathrm{~m})$ & 0.184 & $\frac{\partial M}{\partial \sigma}(\mathrm{N} . \mathrm{m} / \mathrm{Pa})$ & $-0.595 \mathrm{e}-3$ \\
$\frac{\partial L}{\partial \theta}(\mathrm{m} / \mathrm{rad})$ & 0.1159 & $\frac{\partial f}{\partial \sigma}\left(\mathrm{Pa}{ }^{-1}\right)$ & $2.2 \mathrm{e}-10$ \\
$\frac{\partial M}{\partial \theta}(\mathrm{N} . \mathrm{m} / \mathrm{rad})$ & $-8.12 \mathrm{e} 3$ & $\frac{\partial \beta}{\partial \sigma}\left(\mathrm{Pa}{ }^{-1}\right)$ & $3.5 \mathrm{e}-10$ \\
\hline
\end{tabular}

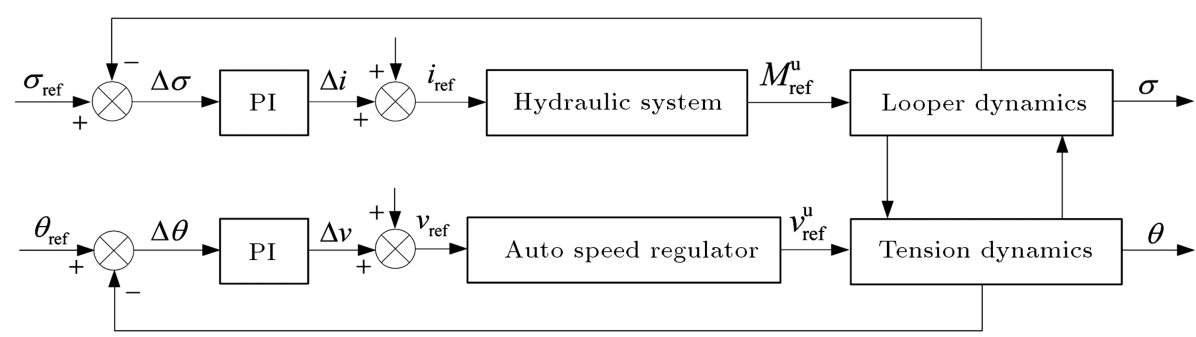

Figure 3. Traditional PI control structure of a hydraulic looper system.

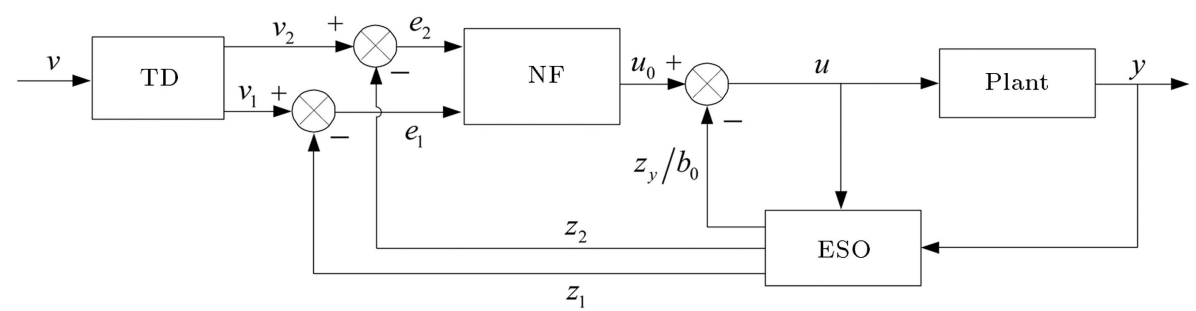

Figure 4. The structure of active disturbance rejection control.

integral transformation, combines control engineering experiences by nonlinear combination of error, and reduces disturbances by error estimation $[18,19]$.

ADRC control system is composed of Tracking Differentiator (TD), Extended State Observer (ESO), and Nonlinear Feedback (NF) law, as shown in Figure 4.

a. Tracking differentiator. Proportional, integral and derivative feedback is based on the past $(I)$, present $(P)$, and future $(D)$ control errors. Nevertheless, most loops are in fact PI because the noise, interfused in input signals or feedback signals, is easily amplified by derivative. The PID equation can be given as follows:

$$
u(k)=\left[K_{p}+\frac{K_{I}}{z^{-1}-1}+K_{D}\left(1-z^{-1}\right)\right] e(k),
$$

where $e(k)$ is control error at the $K$ th instant, $K_{P}$ is proportional coefficient, $K_{I}$ is integral coefficient, $K_{D}$ is differential coefficient, and $u(k)$ is system input signal at the $K$ th instant. Consider a TD model:

$$
\left\{\begin{array}{l}
\nu_{1}^{\prime}=\nu_{2} \\
\nu_{2}^{\prime}=-M \operatorname{sgn}\left[\nu_{1}-\nu_{0}+\left|\nu_{2}\right| \nu_{2} /(2 M)\right],
\end{array}\right.
$$

where $\nu_{1}$ is the tracking result of input signal, $\nu$; $\nu_{2}$ is the differential of $\nu_{1}$; and $M$ is the tracking coefficient. Instead of derivative operation, $\nu_{2}$ can 
be derived from integral operation. It has been found that the integral transformation is beneficial for avoiding differential disaster.

b. Extended state observer. Consider an ESO model:

$$
\begin{aligned}
& \left\{\begin{array}{l}
e=z_{1}-y \\
\dot{z}_{1}=z_{2}-\beta_{01} f a l\left(e, a, \delta_{1}\right) \\
\dot{z}_{2}=z_{3}-\beta_{02} f a l\left(e, a, \delta_{1}\right)+b_{o} u \\
\dot{z}_{3}=-\beta_{03} \text { fal }\left(e, a, \delta_{1}\right)
\end{array}\right. \\
& \text { fal }(x, a, \delta)= \begin{cases}|x|^{\delta} \operatorname{sgn}(x) a & |x| \geq \delta \\
x / \delta^{1-a} & |x| \geq \delta\end{cases}
\end{aligned}
$$

where $z_{1}$ is the tracking result of output signal, $y$; $z_{2}$ is the approximate differential of $z_{1}$; $z_{3}$ is the estimate of disturbance; $\delta$ is the turning point of fal function; $a$ is the nonlinear coefficient; and $\beta_{01}$, $\beta_{02}$, and $\beta_{03}$ are the coefficients of observer.

Note that Eq. (23) is a continual, yet nondifferential, function, which is the arithmetic fit of engineering experience, larger error with less regulated gain, while less error with larger regulated gain.

c. Nonlinear feedback law. A PID controller, which is designed based on the linear combination of proportional, integral, and derivative feedback, is independent of the model of control plant. Because of simple linear combination, there is a limitation in resolving the conflict of fast response and overshoot of control system. Consider an ESO model:

$$
\left\{\begin{array}{l}
e_{1}=\nu_{1}-z_{1} a \\
e_{2}=\nu_{2}-z_{2} a \\
u_{0}=K_{p 1} f a l\left(e_{1}, a, \delta\right)+K_{p 2} f a l\left(e_{2}, a, \delta\right) \\
u=u_{0}-z_{3} / b_{0}
\end{array}\right.
$$

where $e_{1}$ is system error, $e_{2}$ is system differential error, $u_{0}$ is control signal, and $u$ is system input signal. $K_{p 1}$ is proportional gain, and $K_{p 2}$ is differential gain. Control signal $u_{0}$, which inherits the modelindependent characteristic of PID controller and effectively compromises the conflict between fast response and overshoot, is a nonlinear combination of proportional and derivative feedbacks.

\subsection{Application of MADRC technique in a hydraulic looper control system}

For the Double-Input Double-Output (DIDO) hydraulic looper system, the dynamic interaction between loops can also be treated as external disturbance, which can be tracked and estimated by ESO in each main loop. Through the ADRC static decoupling technology, a complex DIDO hydraulic looper system can be converted to two Signal-Input Signal-Output
(SISO) systems: a normal SISO ADRC is then designed for looper height and strip tension closed loops.

To give a quick and transient response to the hydraulic looper system, TD is omitted for the designed system. Besides, traditional ESO and SEF usually consist of nonlinear function structure; the same control result can also be achieved by linear ADRC(LADRC), since the hydraulic looper system can respond very fast; furthermore, LADRC is much easier to realize.

The solution process of ADRC control strategy can be divided into (a) and (b):

(a) ADRC static decoupling technology. For the ADRC decoupling control of hydraulic looper system, a static decoupling compensator should be connected in the front of controlled object; thus, the system matrix (model) can be converted to a diagonal matrix.

The original coupling matrix of hydraulic looper system is a high-order matrix as shown in Eq. (15), which is difficult to achieve in the real system. Therefore, it reduces the order by Pade order-reduced method as follows:

$$
\bar{G}(s)=\left[\begin{array}{ll}
\bar{G}_{v \theta}(s) & \bar{G}_{i \theta}(s) \\
\bar{G}_{v \sigma}(s) & \bar{G}_{i \sigma}(s)
\end{array}\right]
$$

where:

$$
\begin{aligned}
& \bar{G}_{v \theta}(s)=\frac{50.94}{0.0622 s^{2}+0.7415 s+1}, \\
& \bar{G}_{i \theta}(s)=\frac{1.965 e 4}{0.489 s^{2}+1.394 s+1}, \\
& \bar{G}_{v \sigma}(s)=\frac{-13.48}{0.0622 s^{2}+0.7415 s+1}, \\
& \bar{G}_{i \sigma}(s)=\frac{3370 s}{0.489 s^{2}+1.394 s+1} .
\end{aligned}
$$

Due to the time-varying characteristics during rolling process, the static coupling matrix, $\bar{G}(t)$, has great uncertainty; thus, reversible constant matrix, $\bar{G}_{0} \approx \bar{G}(t)_{t=t_{0}}$, is approximately selected in its range, and the approximate error can be classified as disturbance and be expressed as follows:

$$
\begin{aligned}
\bar{G}_{0} & \left.\approx\left[\begin{array}{ll}
\bar{G}_{v \theta}(t) & \bar{G}_{i \theta}(t) \\
\bar{G}_{v \sigma}(t) & \bar{G}_{i \sigma}(t)
\end{array}\right]\right|_{t=t_{0}} \\
& =\left[\begin{array}{ll}
\bar{G}_{v \theta, 0} & \bar{G}_{i \theta, 0} \\
\bar{G}_{v \sigma, 0} & \bar{G}_{i \sigma, 0}
\end{array}\right] .
\end{aligned}
$$

According to the unit matrix synthesis on decoupling control, to decouple the hydraulic looper system, a series static decoupling compensator is designed as follows: 


$$
\left[\begin{array}{l}
x_{v}(s) \\
x_{i}(s)
\end{array}\right]=\left[\begin{array}{ll}
\bar{G}_{v \theta, 0} & \bar{G}_{i \theta, 0} \\
\bar{G}_{v \sigma, 0} & \bar{G}_{i \sigma, 0}
\end{array}\right]\left[\begin{array}{c}
\nu(s) \\
i(s)
\end{array}\right]
$$

where $x_{v}(s)$ is the virtual control variable of looper height closed loop; $x_{i}(s)$ is the virtual control variable of strip tension closed loop.

Then, the Static Decoupling Compensator (SDC) can be expressed as follows:

$$
\begin{aligned}
{\left[\begin{array}{ll}
N_{v \theta} & N_{i \theta} \\
N_{v \sigma} & N_{i \sigma}
\end{array}\right] } & =\bar{G}_{0}^{-1}\left[\begin{array}{ll}
1 & 0 \\
0 & 1
\end{array}\right] \\
& =\frac{1}{\bar{G}_{v \theta, 0} \bar{G}_{i \sigma, 0}-\bar{G}_{i \theta, 0} \bar{G}_{v \sigma, 0}} \\
& {\left[\begin{array}{cc}
\bar{G}_{i \sigma, 0} & -\bar{G}_{i \theta, 0} \\
-\bar{G}_{v \sigma, 0} & \bar{G}_{v \theta, 0}
\end{array}\right] . }
\end{aligned}
$$

As described above, the ADRC static decoupling technology can break through the limit of conventional inverse matrix decoupling method. The technology does not require one to know the exact value of coupling matrix, $\bar{G}(t)$, and its application needs only the roughly estimated value of coupling matrix, $\bar{G}_{0}$. For the approximate error caused by the uncertainty or singularity of $\bar{G}(t)$, ADRC can regard the error as new disturbance, and the automatic disturbance estimate can be realized to compensate for the error.

(b) The design of MADRC system. To facilitate the design of MADRC, the transfer function model of hydraulic looper system should be written into I$\mathrm{O}$ standard form. The approximate errors can be classified as disturbance, and the ADRC of looper height closed loop and strip tension closed loop are then designed as follows:

$$
\begin{aligned}
& \ddot{\theta}=f_{\theta}\left(\theta, \dot{\theta}, d_{\theta}, t\right)+b_{0 \theta} x_{v}(t), \\
& \ddot{\sigma}=f_{\sigma}\left(\sigma, \dot{\sigma}, d_{\sigma}, t\right)+b_{0 \sigma} x_{i}(t),
\end{aligned}
$$

where $f_{\theta}($.$) and f_{\sigma}($.$) are unknown total distur-$ bances; $d_{\theta}$ and $d_{\sigma}$ are unknown external disturbances; $b_{0 \theta}$ and $b_{0 \sigma}$ are tunable parameters.

For Linear Extended State Observer (LESO), it can be designed as follows:

$$
\begin{aligned}
& \left\{\begin{array}{l}
\dot{z}_{1 \theta}=\dot{z}_{2 \theta}-\beta_{01 \theta} \varepsilon_{\theta} \\
\dot{z}_{2 \theta}=\dot{z}_{3 \theta}-\beta_{02 \theta} \varepsilon_{\theta}+b_{0 \theta} x_{v} \\
\dot{z}_{3 \theta}=-\beta_{03 \theta} \varepsilon_{\theta} \\
\varepsilon_{\theta}=z_{1 \theta}-\theta
\end{array}\right. \\
& \left\{\begin{array}{l}
\dot{z}_{1 \sigma}=\dot{z}_{2 \sigma}-\beta_{01 \sigma} \varepsilon_{\sigma} \\
\dot{z}_{2 \sigma}=\dot{z}_{3 \sigma}-\beta_{02 \sigma} \varepsilon_{\sigma}+b_{0 \sigma} x_{i} \\
\dot{z}_{3 \sigma}=-\beta_{03 \sigma} \varepsilon_{\sigma} \\
\varepsilon_{\sigma}=z_{1 \sigma}-\sigma
\end{array}\right.
\end{aligned}
$$

By selecting suitable tunable parameters, $\beta_{01 \theta}$, $\beta_{02 \theta}, \beta_{03 \theta}, b_{0 \theta}, \beta_{01 \sigma}, \beta_{02 \sigma}, \beta_{03 \sigma}, b_{0 \sigma}$, the designed LESO can quickly and accurately track the looper system states and estimate the total disturbances of hydraulic looper system:

$$
\begin{aligned}
& z_{1 \theta} \rightarrow \theta, z_{2 \theta} \rightarrow \dot{\theta}, z_{3 \theta} \rightarrow f_{\theta}(\cdot), \\
& z_{1 \sigma} \rightarrow \sigma, z_{2 \sigma} \rightarrow \dot{\sigma}, z_{3 \sigma} \rightarrow f_{\sigma}(\cdot) .
\end{aligned}
$$

As shown in Eqs. (33) and (34), it is clear that $z_{3 \theta}$ and $z_{3 \sigma}$ are able to estimate the total disturbance of hydraulic looper system. Also, $f_{\theta}($.$) and f_{\sigma}($.$) can$ be rejected if real-time compensation terms $-z_{3 \theta} / b_{0 \theta}$ and $-z_{3 \sigma} / b_{0 \sigma}$ are contained in the control law; thus, the control laws of looper height closed loop and strip tension closed loop are designed as follows:

$$
\begin{aligned}
& x_{v}=\left(x_{0 v}-z_{3 \theta}\right) / b_{0 \theta}, \\
& x_{i}=\left(x_{0 i}-z_{3 \sigma}\right) / b_{0 \sigma} .
\end{aligned}
$$

By substituting Eq. (35) into Eq. (29) and substituting Eq. (36) into Eq. (30), the plant of hydraulic looper system can be reduced approximately to the unit gain cascade plant as follows:

$$
\begin{aligned}
& \ddot{\theta}=f_{\theta}\left(\theta, \dot{\theta}, d_{\theta}, t\right)-z_{3 \theta}+x_{0 v} \approx x_{0 v}, \\
& \ddot{\sigma}=f_{\sigma}\left(\sigma, \dot{\sigma}, d_{\sigma}, t\right)-z_{3 \sigma}+x_{0 i} \approx x_{0 i} .
\end{aligned}
$$

Then, it will be easy to design a perfect control law by state error feedback. Inheriting from PID based on error-based feedback control, controlled inputs $x_{0 v}$ and $x_{0 i}$ often employ the nonlinear combinations to simulate manual's control strategy that has an intelligent function in a sense. To satisfy rapid requirements of the hydraulic looper system and ensure ease of use, the Linear State Error Feedback (LSEF) control laws are designed as follows:

$$
\begin{aligned}
& x_{0 \theta}=k_{p \theta} e_{\theta}-k_{d \theta} z_{2 \theta}, \\
& x_{0 \sigma}=k_{p \sigma} e_{\sigma}-k_{d \sigma} z_{2 \sigma},
\end{aligned}
$$

where $e_{\theta}=\theta_{\text {ref }}-z_{1 \theta}, e_{\sigma}=\sigma_{\text {ref }}-z_{1 \sigma}$, and $\theta_{\text {ref }}$ is the reference of looper height; $\sigma_{\text {ref }}$ is the reference of strip tension; $k_{p \theta}, k_{d \theta}, k_{p \sigma}$, and $k_{d \sigma}$ are the adjusted gain parameters.

The structure of MADRC for the hydraulic looper system is shown in Figure 5, and SDC is designed as Eq. (28); LESO can compensate for real-time unknown total disturbances $f_{\theta}($.$) and f_{\sigma}($.$) based on roll velocity$ $\nu$, control current $i$, strip tension $\sigma$, and looper angle $\theta$; LSEF can generate control variables $x_{0 \theta}$, and $x_{0 \sigma}$ based on the assignment for state error feedback and the compensation for disturbance estimation. 


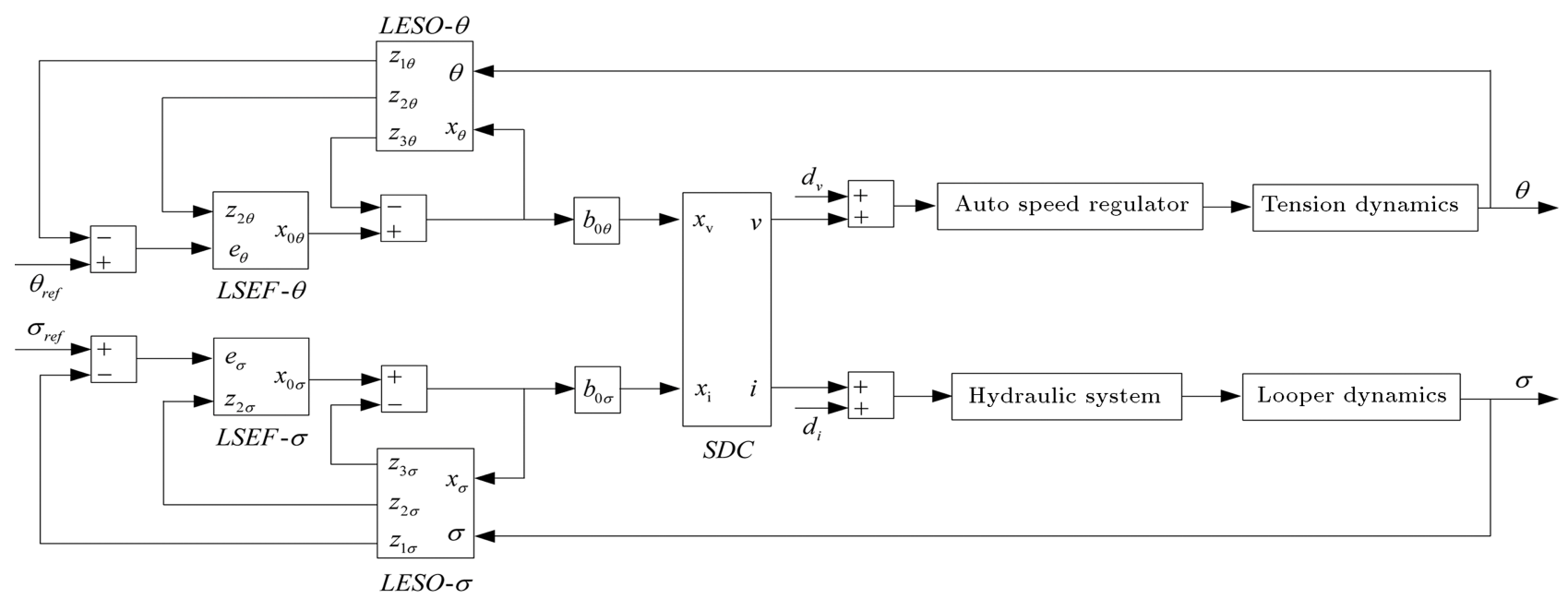

Figure 5. The structure of multivariable active disturbance rejection control for a hydraulic looper system.

As shown in Figure 5, instead of designing a decoupling subsystem in the MADRC control system and considering the static decoupling mismatch caused by model perturbation, the uncertainty of controlled object and unknown external disturbance can be regarded as total disturbance, which can be compensated by LESO so that indirect decoupling control and active disturbance rejection control can be achieved.

\section{Simulation experiments}

It should be noted that the application of the new control method described herein to an actual installation is an extremely expensive task that is well beyond the scope of this paper. This is because, in most cases, interrupting production on a multi-million dollar manufacturing process, such as a tandem hot strip mill, to test and verify a new large scale control strategy is virtually impossible mostly due to the very high manufacturing costs and is usually not allowed by the mill owner. Thus, the only reasonable method for verifying the performance of a controller in this scope is by simulation. Using the working and control principles of a hydraulic looper in a hot rolling mill, the control effects of PI and MADRC controllers were compared by using MATLAB/Simulink software.

Based on PI and MADRC control schemes developed above, the structures of PI and MADRC controllers used in simulations can be expressed as follows:

$$
\begin{aligned}
& C_{P I}(s)=P+I\left(\frac{1}{s}\right), \\
& C_{M A D R C}(s)=\sum_{i=1}^{n} \beta_{i} f a l\left(e_{i}, a, \delta\right) .
\end{aligned}
$$

The parameters of PI and MADRC controllers used in simulations are listed in Table 2.
Table 2. List of controller parameters.

\begin{tabular}{ccccccc}
\hline Items & \multicolumn{1}{c}{ PI } & \multicolumn{5}{c}{ MADRC } \\
\hline & $P_{v}^{\prime}$ & 1.25 & $\beta_{01 \theta}$ & 150 & $\beta_{01 \sigma}$ & 120 \\
& & & $\beta_{02 \theta}$ & 350 & $\beta_{02 \sigma}$ & 4800 \\
& & & & & & \\
Controller & $I_{v}^{\prime}$ & 0.73 & $\beta_{03 \theta}$ & 1200 & $\beta_{03 \sigma}$ & 30000 \\
parameters & & & & & & \\
& $P_{i}^{\prime}$ & $5.22 \mathrm{e}-5$ & $b_{0 \theta}$ & 70 & $b_{0 \sigma}$ & 50 \\
& & & $k_{p \theta}$ & 46.9 & $k_{p \sigma}$ & 0.24 \\
& & & & & & \\
& $I_{i}^{\prime}$ & 8.23 & $k_{d \theta}$ & 23.3 & $k_{d \sigma}$ & 1.66 \\
\hline
\end{tabular}

\subsection{Simulations for comparing the step responses}

The simulations were carried out as in the following steps. A step testing signal with amplitude of $0.02 \mathrm{rad}$ was added to the initial value of the looper angle at $t=$ $5 \mathrm{~s}$, and the simulation results are shown in Figure 6 . A step testing signal with amplitude of $1.0 \mathrm{MPa}$ was added to the initial value of the looper angle at $t=5 \mathrm{~s}$, and the simulation results are shown in Figure 7.

The dynamic characteristic parameters of PI and MADRC controllers were calculated, and the results are presented in Table 3.

As shown in Table 3 above, PI controller gives an angle rising time of $0.93 \mathrm{~s}$, a tension rising time of $0.46 \mathrm{~s}$, an overshoot angle of $0.16 \%$, and an overshoot tension of $0.12 \%$. The proposed MADRC controller produces a lower tension rising time of just $0.04 \mathrm{~s}$ and an overshoot angle of only $0.12 \%$. The dynamic characteristic parameters of both controllers are acceptable in a hydraulic looper system. Furthermore, because of the ADRC static decoupling technology and ESO 
Table 3. Comparison of dynamic characteristics.

\begin{tabular}{ccccc}
\hline Items & Controller & Rising time & Overshot & Range \\
\hline \multirow{2}{*}{ Step change in angle } & PI & $0.93 \mathrm{~s}$ & $0.16 \%$ & $0.135 \mathrm{MPa}$ \\
& MADRC & $0.12 \mathrm{~s}$ & $0.12 \%$ & $0.015 \mathrm{MPa}$ \\
& & & & \\
Step change in tension & PI & $0.46 \mathrm{~s}$ & $15.4 \%$ & $0.009 \mathrm{rad}$ \\
& MADRC & $0.04 \mathrm{~s}$ & $0.3 \%$ & $0.0005 \mathrm{rad}$ \\
\hline
\end{tabular}

dynamic decoupling technology in the designed control system, the tension fluctuation is $0.015 \mathrm{MPa}$, and the angle fluctuation is $0.0005 \mathrm{rad}$, which is much smaller than that of the PI controller.

\subsection{Simulation for comparing the disturbance rejection performances}

In an actual hot strip rolling process, there is a mutual interaction between the automatic gauge control system and the hydraulic looper control system. In the hydraulic looper system, the main disturbance comes from changes in mass flow. We added changes in the roll gap, regarded as the disturbance signals, to the strip tension closed loop, and the fluctuation in entry thickness and temperature is replaced approximately by Gaussian noise signals.

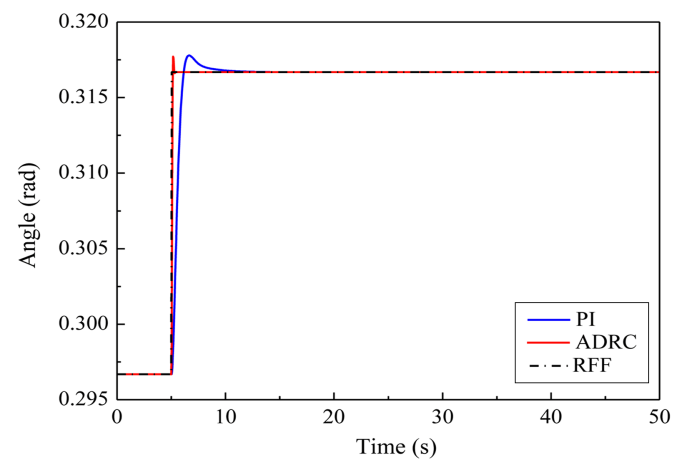

A sinusoidal roll gap disturbance, $\Delta S_{a}$, with frequency of $0.5 \mathrm{~Hz}$ and amplitude of $0.05 \mathrm{~mm}$ was added to the initial value of the roll gap, and the simulation results are shown in Figure 8. A step testing signal with amplitude of $0.05 \mathrm{~mm}$ was added to the initial value of the roll gap at $t=5 \mathrm{~s}$, and the simulation results are shown in Figure 9.

As shown in the above simulation results, under the same disturbance situation, MADRC controller can achieve control the effect of looper angle and strip tension better than PI controller may. The MADRC has the high disturbance rejection performance, because this control strategy introduces LESO into the control system so that the total disturbance caused by the uncertainty of controlled object and unknown external disturbance can be compensated.

Figure 6. Response curves of looper angle step disturbance.
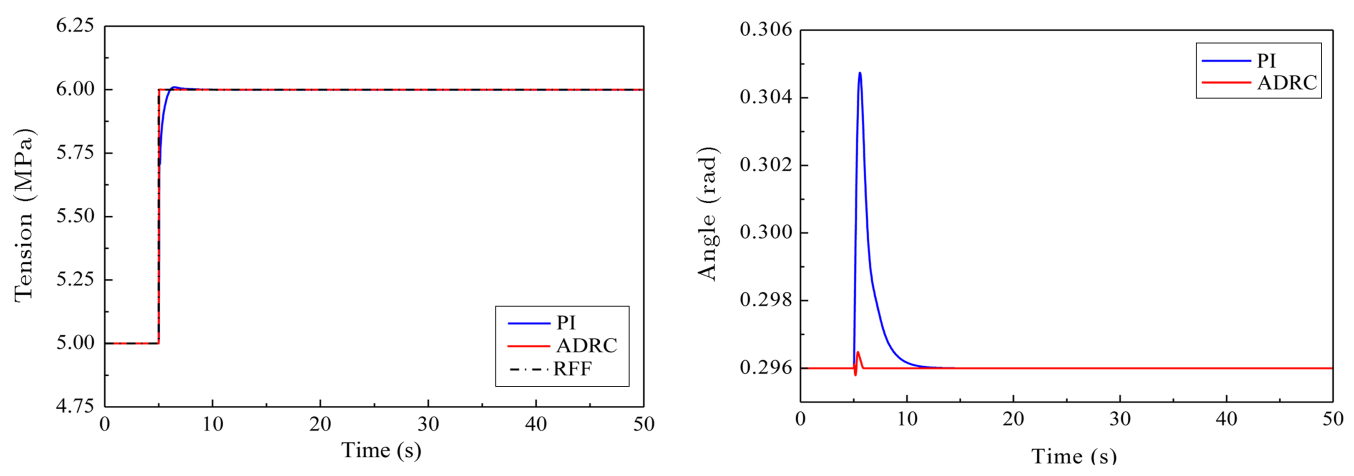

Figure 7. Response curves of strip tension step disturbance. 

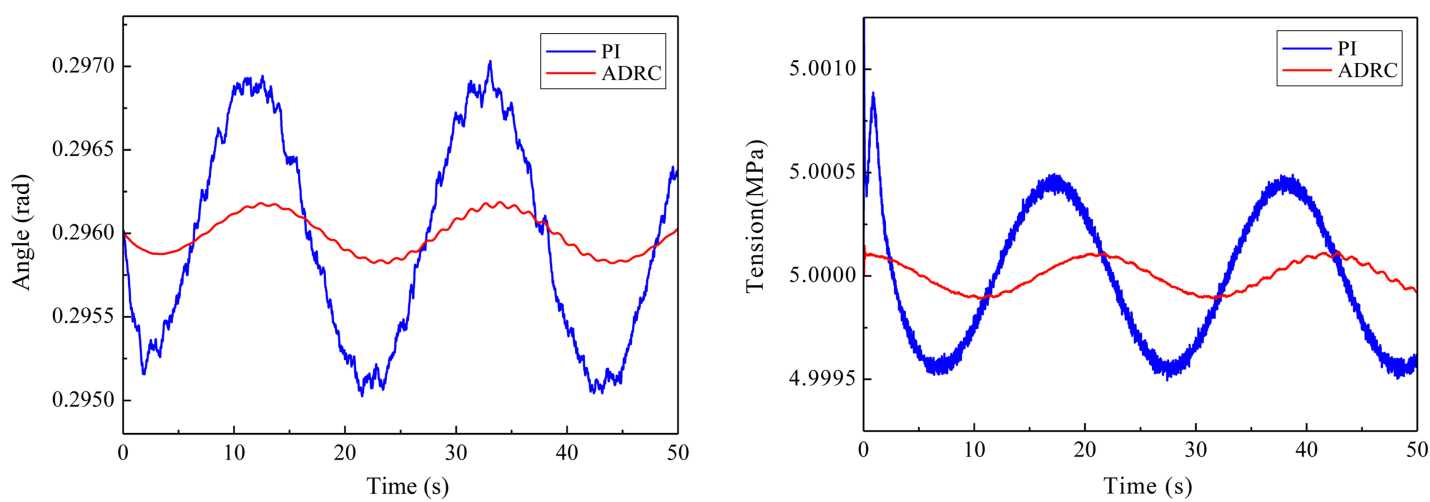

Figure 8. The outputs of hydraulic looper system in response to roll gap sine disturbance.
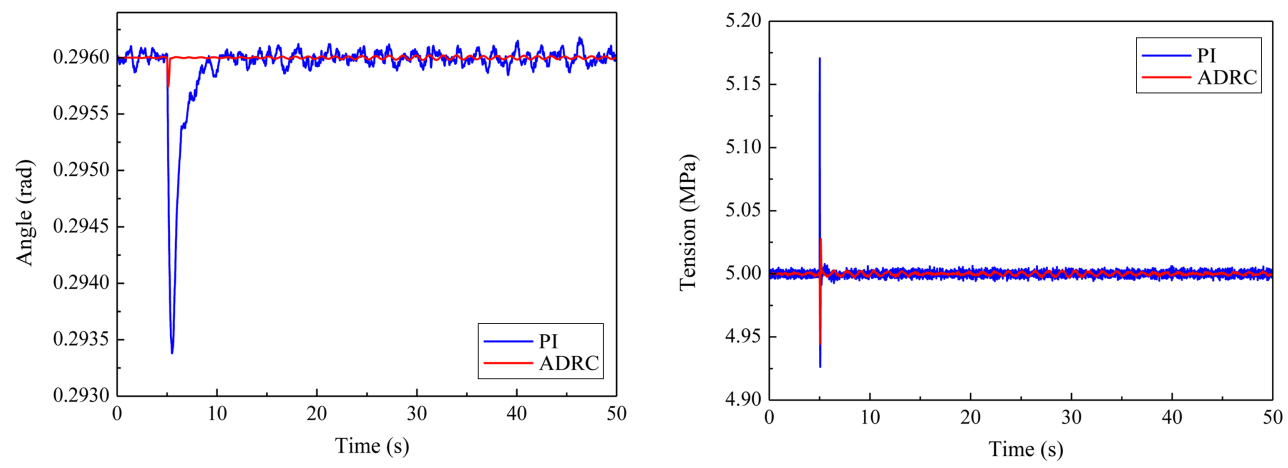

Figure 9. The outputs of hydraulic looper system in response to roll gap step disturbance.

\subsection{Simulation with varying rolling process parameters}

In actual hot strip mill production, various strip specifications are rolled. Thus, the hydraulic looper system must be robust enough to ensure the quality of strips. In this simulation, the effect of different CrossSectional Areas (CSA) was investigated under both PI and MADRC controllers.

A step disturbance with amplitude of $0.02 \mathrm{rad}$ was added to the initial value of the looper angle at $t=1 \mathrm{~s}$. The simulation results are shown in Figures 10 and 11 and Table 4. As the cross-sectional areas enlarge, the overshoot angle of both controllers remains fairly good, although the rising time of PI controller increases to $731.9 \mathrm{~ms}$, which is unacceptable in the hydraulic looper system. In contrast, the rising time of MADRC controller remains below $70 \mathrm{~ms}$. When the crosssectional areas of the strip become smaller, the rising time of both controllers decreases. However, for PI controller, the minimum rising time is $731.8 \mathrm{~ms}$, which does not meet the dynamic response requirements of the hydraulic looper system. However, MADRC controller achieves a rising time of just $63.8 \mathrm{~ms}$ and an overshoot angle of $12.17 \%$. These levels are acceptable for hydraulic looper systems. Additionally, the tension overshoot of MADRC controller is less than $0.35 \mathrm{MPa}$ in all cases, which is less than that of PI controller.

Table 4. Dynamic characteristic parameters achieved with looper angle step disturbance.

\begin{tabular}{|c|c|c|c|c|c|c|c|c|}
\hline \multirow[t]{2}{*}{ Parameters } & \multicolumn{2}{|c|}{$\mathrm{CSA}=1448 \mathrm{~mm}^{2}$} & \multicolumn{2}{|c|}{$\mathrm{CSA}=2230 \mathrm{~mm}^{2}$} & \multicolumn{2}{|c|}{$\mathrm{CSA}=4952 \mathrm{~mm}^{2}$} & \multicolumn{2}{|c|}{$\mathrm{CSA}=7825 \mathrm{~mm}^{2}$} \\
\hline & PI & MADRC & PI & MADRC & PI & MADRC & PI & MADRC \\
\hline Angle rising time (s) & 731.8 & 63.8 & 731.5 & 64.2 & 732.8 & 65.7 & 731.9 & 65.5 \\
\hline Angle overshot (\%) & 13.52 & 12.17 & 13.67 & 11.98 & 13.28 & 12.08 & 13.41 & 12.32 \\
\hline $\begin{array}{c}\text { Tension fluctuation } \\
\text { range }(\mathrm{MPa})\end{array}$ & 0.652 & 0.332 & 0.538 & 0.318 & 0.428 & 0.224 & 0.327 & 0.183 \\
\hline
\end{tabular}



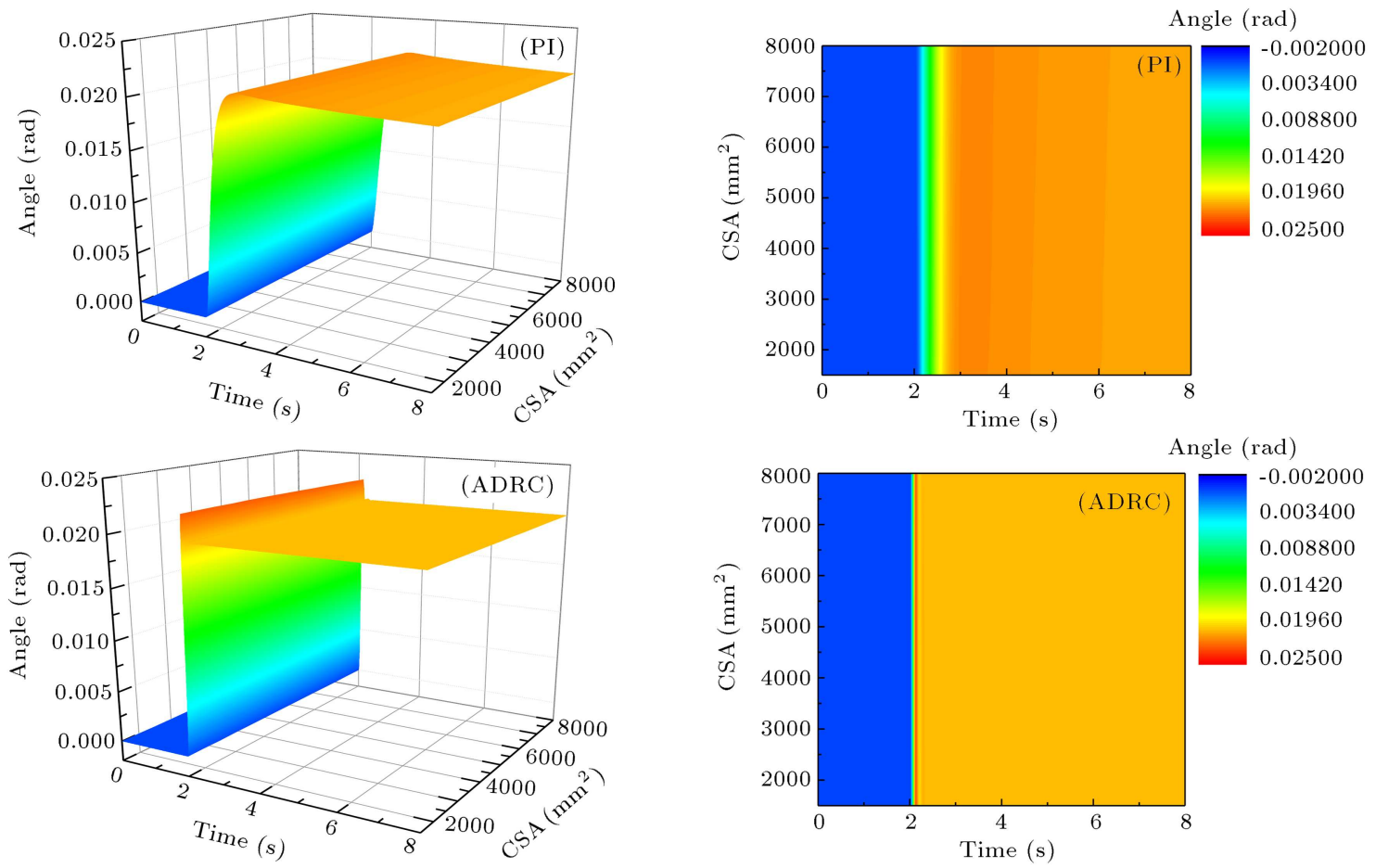

Figure 10. Response curves of looper angle step disturbance with varying strip cross-sectional areas.
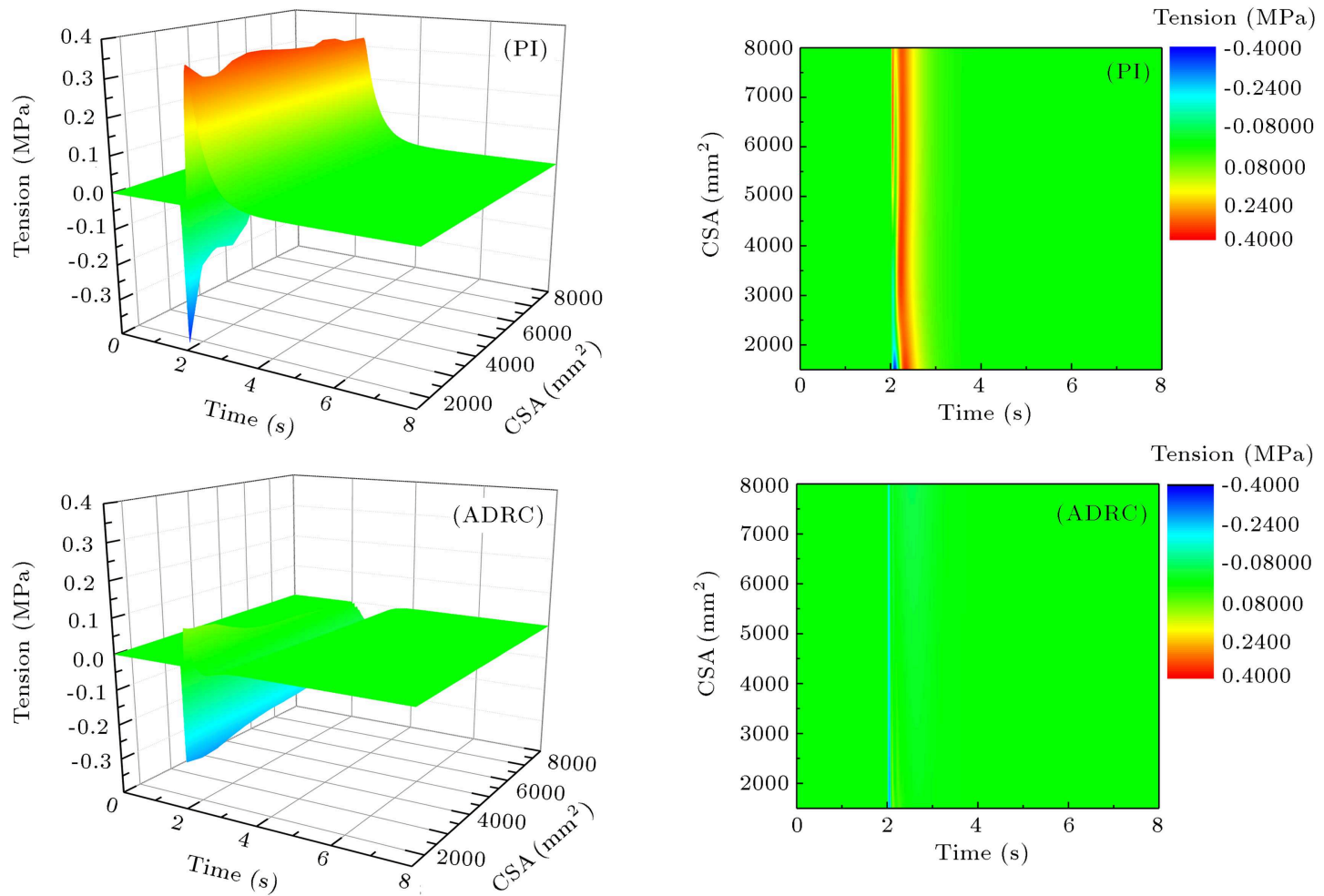

Figure 11. Response curves of strip tension step disturbance with varying strip cross-sectional areas.

According to the above results, it is clear that both controllers with model matching can achieve good control effects. When the system is disturbed or the rolling process parameters change, the disturbance rejection and robustness of PI controller are degraded, and large overshoots or long rising times appear. However, the hydraulic looper control system based on the proposed MADRC controller is relatively insensitive to disturbances and rolling process parameter changes, exhibiting strong disturbance rejection and robustness. 


\section{Conclusions}

1. Based on the generation mechanism of strip tension and the force conditions of a hydraulic looper, a dynamical mathematical model of a hydraulic looper system was established. MADRC controller that allows active disturbance rejection control was designed for the established model;

2. Simulation platforms for MADRC and PI control systems were developed using MATLAB/Simulink. A comparison of the step response and disturbance rejection performance with model matching was carried out. The simulation results showed that, under the condition that the disturbance comes from roll gap, MADRC controller could achieve better dynamic performance with higher levels of control;

3. Comparison experiments of different cross-sectional areas of strip were carried out. The simulation results showed that the overshoot and rising time of PI controller increased beyond the levels accepted by hydraulic looper systems. In the case of MADRC controller, the overshoot angle remained less than $12.5 \%$, and the rising time was less than $70 \mathrm{~ms}$. The tension fluctuation range was less than $0.35 \mathrm{MPa}$ in all scenarios. Thus, the control performance of the proposed DMC controller was better than that of PI controller.

\section{References}

1. Sansal, K.Y., Huang, B., and Forbes, J.F. "Dynamics and variance control of hot mill loopers", Control Engineering Practice, 16(1), pp. 89-100 (2008).

2. Sansal, K.Y., Forbes, J.F., and Huang, B. "Dynamic modelling and simulation of a hot strip finishing mill", Applied Mathematical Modelling, 33(7), pp. 3208-3325 (2009).

3. Choi, I.S., Rossiter, J.A., and Fleming, P.J. "Looper and tension control in hot rolling mills: a survey", Journal of Process Control, 17(6), pp. 509-521 (2007).

4. Kazuy, A., Takashi, K., and Nobukai, N. "Hot strip mill tension-looper control based on decentralization and coordination", Control Engineering Practice, 8(3), pp. 337-344 (2000).

5. Hearns, G. and Grimble, M.J. "Robust multivariable control for hot strip mills", ISIJ International, 40(10), pp. 995-1002 (2000).

6. Cheng, C.C., Hsu, L.Y., and Chen, Z.S. "Precise looper simulation for hot strip mills using an autotuning approach", The International Journal of Advanced Manufacturing Technology, 27(5), pp. 481-487 (2006).

7. Li, Z.J., Shi, X., Liu, H.P., and Yang, W.D. "Modeling and control of hydraulic looper multivariable systems", Journal of University of Science and Technology Beijing, 32(10), pp. 1353-1359 (2010).
8. Riccardo, F., Francesco, A.C., and Thomas. P. "Friction compensation in the interstand looper of hot strip mills: a sliding- mode control approach", Control Engineering Practice, 16(2), pp. 214-224 (2008).

9. Zhong, Z.Z., Wang, J.C., and Zhang, J.M. "Loopertension sliding mode control for hot strip finishing mills", Journal of Iron and Steel Research International, 19(1), pp. 23-30 (2012).

10. Wang, H.W., Jing, Y.W., and Yu, C. "Guaranteed cost sliding mode control for looper-tension multivariableuncertain systems", Nonlinear Dynamics, 80(1-2), pp. 39-50 (2015).

11. Zhong, Z.Z. and Wang, J.C. "Looper-tension almost disturbance decoupling control for hot strip finishing mill based on feedback linearization", IEEE Transactions on Industrial Electronics, 58(8), pp. 3668-3677 (2011).

12. Pittner, J. and Simaan, M.A. "A useful control model for tandem hot metal strip rolling", IEEE Transactions on Industry Applications, 46(6), pp. 2251-2258 (2010).

13. Wang, L.J., Tong, C.N., and Li, Q. "Practical active disturbance rejection solution for monitoring automatic gauge control system with large time-delay", Control Theory and Apply, 29(3), pp. 368-374 (2012).

14. Zou, J., Fu, X., and Yang, H.Y. "Active disturbance rejection control for hydraulic width control system for rough mill", Journal of Zhe Jiang University Sci A, 8(9), pp. 1429-1434 (2007).

15. Huang, Y., Xu, K., Han, J., and Lam, J. "Flight control design using extended state observe and nonsmooth feedback", Proceedings of the 2001 IEEE Conference on Decision and Control, Florida, USA, pp. 223-228 (2001).

16. Su, J.B. and Qiu, W.B. "Robotic calibration-free handeye coordination based on auto disturbances rejectioncontroller", Acta Automatica Sinica, 29(2), pp. 161167 (2003).

17. Dong, L., Zheng, Q., and Gao, Z. "A novel controller design for electric power assist steering systems", Journal of Intelligent Control and Systems, 8(11), pp. 1871-1878 (2008).

18. Wang, Y., Zhang, Z., and Qin, X.Q. "Modeling and control for hydraulic transmission of unmanned ground vehicle", Journal of Central South University of Technology, 21(1), pp. 124-129 (2014).

19. John, P. and Marwan, A.S. "Improvement in control of the tandem hot strip mill", IEEE Transactions on Industry Applications, 49(5), pp. 1962-1970 (2013).

20. Jiao, X.H., Shao, L.P., and Peng, Y. "Adaptive coordinated control for hot strip finishing mills", Journal of Iron and Steel Research International, 18(4), pp. 36-43 (2010).

21. Okada, M., Murayama, K., and Urano, A. "Optimal control system for hot strip finishing mill", Control Engineering Practice, 2(8), pp. 1029-1034 (1998). 
22. Han, J.Q. "Linear and nonlinear of feedback system", Control Decision, 3(1), pp. 27-32 (1998).

23. Han, J.Q. "Auto disturbances rejection controller and its applications", Control Decision, 13, pp. 19-23 (1998).

24. Gao, B.W., Shao, J.P., and Yang, X.D. "A compound control strategy combining velocity compensation with ADRC of electro-hydraulic position servo control system", ISA Transactions, 53(6), pp. 1910-1918 (2014).

25. Zhao, Z.L. and Guo, B.Z. "On active disturbance rejection control for nonlinear systems using timevarying gain", European Journal of Control, 23, pp. 62-70 (2015).

\section{Biographies}

Fang Chen Yin received the BSE degree in Material Processing from Shenyang University of Technology, China, and the MSE and PhD degrees in Material Processing from the Northeastern University, China in
2013 and 2017, respectively. He is currently a Lecturer at the HuaQiao University. He has been involved in developing, designing, and commissioning various areas of several rolling mill projects nationally and internationally.

Cai Zhi Wang received the BSE degree in Mechanical Manufacturing from Anhui University of Science and Technology, China in 2017. He is currently a Postgraduate Student at the HuaQiao University. He is currently working on active disturbance control strategy.

Hui Shao received the BSE degree in Control Engineering from Northeast Electric Power University, China and the MSE and PhD degrees in Material Processing from the Ganshu University of Technology and Shanghai Jiaotong University, China in 2001 and 2006, respectively. He is currently an Associate Professor at the HuaQiao University. He is currently working on modern control theory. 